\title{
CAL and FE: a Welsh perspective
}

\author{
*Antje Cockrill, *Cliona O'Neill, *Eberhard Bischoff and **David Finch \\ *University of Wales Swansea \\ **FFORWM \\ email:a.cockrill@swan.ac.uk
}

The core of this paper is the result of an investigation into the use of computer-aided learning (CAL) in further education ( $F E$ ) colleges in Wales: All institutions surveyed used $C A L$ to some extent in teaching or learning, and academic staff were in some way involved in producing CAL materials in almost all. However, student numbers were found not to be a good indicator of the degree to which CAL is used. Furthermore, only twothirds of colleges approached claimed to have received any external funding for $C A L$ and distance learning involved only a small proportion of FE students. Nevertheless, the general conclusion drawn is that all FE colleges in Wales use ICTs (information and communication technologies) extensively, although there are significant differences in the equipment/student ratios, and there appears to be little strategic college-wide planning concerning such developments.

\section{Introduction}

The increasing usage in recent years of trendy, but often ill-defined, terms such as 'lifelong learning' (see Edwards, Raggatt, Harrison, McCollum and Calder, 1998), 'the learning society' (for example, National Grid for Learning, http://www.dfee.gov.uk/grid/challengel govern.htm), or 'learning country' (for example, Welsh Office, 1998; ETAG, 1999) indicates the importance that both the public and private sectors attach to the establishment of a learning culture. This has included the recognition that, in order to achieve such a culture, access to learning must be made easier and existing barriers removed. Edwards and his coauthors (1998) maintain that most experts see lifelong learning as a rallying cry, rather than a specific policy. This statement holds true for many similar slogans, but what they have in common is 'the power to unite various stakeholders around the need for change, because it has emerged as a response to today's challenges' (Edwards et al., 1998). 
Stephen Byers, Secretary of State for Trade and Industry, emphasized last year that while the first industrial revolution was based on investment in capital and machinery, 'the revolution we are going through now requires investment in human capital - skills, learning and education' (Mansion House speech, 2 February 1999, quoted in Future Unit, 1999). This revolution has led to a demand for increased flexibility of learning, and in particular a demand for a significant increase in the numbers participating in further education. Digital broadcasting, the Internet and other computerized telecommunications technologies should have an enormous potential in teaching and learning within post-16 education. In particular, they should enable those who in the past missed out on such education, for whatever reason, to take in later life the steps they might have taken earlier had the opportunities been available.

Nowhere is this more poignantly evident than in Wales, with its long-established perception of education as the principal means of escaping industrial poverty, but where geographical isolation and a lack of public-transport infrastructure has meant that entire communities have lacked easy physical access to educational provision beyond secondary school (see for example, the location of communities such as Glyncorrwg, Croeserw and Cymmer in the Upper Afan Valley). The new flexible learning methods incorporating information and communications technologies (ICTs) and thus computer-aided learning (CAL) would seem to be tailor-made for Wales. For the purposes of this paper, CAL has been defined as the use of computer-based materials and tools (such as CD-ROMs, the Internet, intranets, PowerPoint) in learning and teaching.

In many respects, it is the FE sector that should be taking the lead here. Widened access to university education is a legitimate and desirable priority, but an approach that aims to access and to widen Wales's educational potential in its entirety is doubtless the one that should be the most vigorously explored. New information technologies allow flexible access to expertise and materials (see Furnell, Evans, Phippen and Abu-Rgheff, 1999); facilitate support for part-time students and for students who are absent for parts of courses; encourage different learning styles (see, for example, Johnston, 1997; Gain and De Cicco, 1999); there is potentially high audience involvement through interaction (see Jih, 1996); and they help reduce the problems of high staff-student ratios. In addition; courses can be easily updated or adapted; course material can be personalized (Sargant, 1997); information is available at all times, both on-site and from isolated locations; and instructors may have a reduced work-load once the material is online. A number of studies have also found that interactive training using computer-aided learning increases retention (see, for example, Gunasekaran and Love, 1999); and some authors maintain that learning gains through the use of digital multimedia are 56 per cent greater, consistency of learning 50 to 60 per cent better, learning curves 60 per cent faster and retention 25 to 50 per cent greater (see Burgess, 2000).

Although there is a tendency for some researchers and practitioners alike to disregard potential drawbacks of CAL, it is patent that, for a region such as Wales, flexible learning incorporating the use of CAL presents constructive and highly functional prospects which simply cannot be ignored, especially by FE with its mission of wide access. However, the implementation and use of CAL is not without (as yet) unsolved problems, such as lack of direct interaction between student and academic staff and/or other students (Furnell, Onions, Bleimann, Gojny, Knahl, Rder and Sanders, 1998), large file sizes for multimedia 
data, leading to long download times; problems of compatibility; serious snags associated with intellectual property rights; hidden problems of staff time (Eisenberg, 1998); costs if student numbers are not sufficiently high; and security issues (Furnell et al, 1998). In view of these problems, Sargant (1997) summarizes the opportunities offered by new technologies as 'the challenge ... to harness the most appropriate technology to suit the teaching and learning purpose'.

\section{Welsh FE and ICT initiatives}

In 1996, the Welsh FE sector was characterized by limited usage of and expertise in CAL and ICTs generally, and a distinct lack of integration of its systems (Lockitt, 1999). A Further Education Funding Council for Wales (FEFCW) initiative known as FE-NET was launched in 1995/6 to network all FE colleges in Wales and to provide standard connection and equipment (FEFCW, 1995). Using the Joint Academic Network (JANET), this gave all Welsh FE colleges access to the Internet, as well as an intra-college communication channel. An accompanying project management tool, FE-NET in Action, was developed to raise awareness of the potential of ICT networks for learning and teaching in FE.

Running parallel with this was the setting up of the QUILT (Quality in Information and Learning Technology) Wales project. QUILT Wales, which targeted college-based development programmes, staff training and the design and production of flexible and open learning materials, emerged out of the recommendations of the Higginson Report (the report of the English Further Education Funding Council's Learning and Technology Committee - FEFC, 1996), and a joint FFORWM (the Welsh Colleges' Forum) and Further Education Development Agency (FEDA) conference.

In 1996, colleges had $64 \mathrm{kbps}$ connections, and following an interim upgrade to $384 \mathrm{kbps}$ in 1999 (equivalent to ISDN-6), all colleges will have 2 mbps connections by 2001 . To the university sector, the existing connection, when compared with the very high-speed links actually offered by JANET, may seem inordinately slow for use with multimedia data, but ISDN-6 allows for high-quality audio-graphics, and even video of an acceptable quality (Jacobs and Rodgers, 1997; 1998).

By 1998, almost all FE colleges had made extensive use of their connections to JANET indicating the colleges' readiness to incorporate CAL into the curriculum. The Internet was being used for email, data exchange and searching for information, but with increasing direct use in teaching and learning. Between 1996 and 1999, 21 colleges in Wales had been involved in one or more QUILT college-based projects (FEFCW, 1999).

Strategic planning to support the infrastructural framework continues with FEFCW commissioning two recent reports: a video networking and conferencing study (UKERNA, 1999), and the Becta survey into information and learning technology provision, access and policy (Becta, 2000). The major focus of the Becta survey was to assess levels of computer hardware and ILT (Information and Learning Technology) infrastructures within the Welsh FE sector.

Given such official activity and proposals aimed at increasing both the connectivity of the Welsh FE sector and the general use of new technologies for learning and teaching, and 
given the perceived climate of opportunities for such developments, we conducted a sample survey intended to establish the extent to which initiatives had so far produced results.

\section{The survey}

Initial contact with all FE colleges in Wales was made by telephone. We attempted to identify one person in each college associated with CAL, who was then sent a written survey. The questionnaire contained ten main questions, some of which included queries on a range of topics (e.g. use of CAL, involvement of academic staff in the production of CAL material, Web-based learning, distance learning, numbers of computers, printers, scanners etc., numbers of support staff, availability of equipment to different types of students). These questions were designed to determine the degree to which CAL was used, both in classes and for unsupervised use; the amount of distance learning carried out by the colleges and the use of CAL for this purpose; the availability of learning materials on an intranet or the Internet; the range and quantity of ICT equipment in the FE colleges; and the numbers of support staff available.

The principal limitation of the methodology was the difficulty in obtaining a full complement of responses, but twelve valid completed questionnaires were obtained and used. The respondents were either senior college officers or managers with responsibilities in the area of CAL. Although there might have been some skewing due to the fact that the questionnaires were answered by individuals in a similar position, the authors anticipated that this would also facilitate comparison of the results and help reduce some of the errors that might otherwise arise from different perceptions of staff holding a variety of positions. The colleges varied in size: both the smallest and the largest FE colleges in Wales (as measured by student numbers) were included, and all areas of Wales were represented.

\section{Results and discussion}

Approximately a third of colleges claimed to have no central co-ordinator for CAL, since the functions of a CAL co-ordinator are often distributed among a number of individuals in Welsh FE colleges (Information and Learning Technology Manager, Flexible Learning Centre Manager, Learning Resources Centre Manager or other similar title). For example, Systems Resources (CAL learning materials) and curriculum development may have different managers, with CAL materials being obtained by, for instance, both the Library/Learning Resources Manager and curriculum managers. Indeed, it appears that a recent proliferation of CAL-related projects has increased fragmentation to the point where, in some colleges, no one individual seems to have a global view of what is happening in this regard, implying that potentially useful experiences are not shared in a structured way.

Welsh FE is, of course, not alone in such a situation, either with respect to FE colleges in other parts of the UK or in post-16 education generally. The origin of CAL in Welsh FE colleges is commonly department-specific, this state of affairs often being historical rather than based on current trends and needs. The results of our survey seem to point to a particularly acute problem in this respect with regard to Welsh FE colleges, which reflects the situation in Wales throughout all education sectors except higher education (which is well served), and public use of ICTs generally. The Spectrum Regional Survey commissioned by the UK government (Spectrum, 1999) into the general use of ICT (which 
is undoubtedly the most reliable source of data in this area) shows Wales to be well behind the other UK regions, not only in terms of a comparison with the UK average, but also in terms of a league table of the twelve regional UK divisions. Wales only marginally betters the bottom region, Northern Ireland, which of course has special problems. One of the principal reasons given for this situation is the lack of an overarching strategy in Wales.

Our survey revealed that where a co-ordinator existed in an FE college, the position was often attached to some form of division of support or learning service, although in some colleges the job of co-ordinator was located in a specific subject or course division. Others apparently had no form of organization of CAL whatsoever. The clear implication of this is that Wales should follow the lead of the English FEFC: Welsh colleges need to produce and implement - ILT (Information and Learning Technology) Implementation Plans on the lines of those that have been produced and partly implemented in England (FEFC 1999a; 1999b; 1999c). The FEFCW is preparing for something similar by having commissioned the Becta study referred to above. The recent bulletin entitled Further Education Funding in Wales: 2000-2001 (FEFCW, 2000) stresses that preliminary outcomes of the survey indicate a clear need for funding to be made available for upgrading and increasing FE institutions' computer stock and developing local area networks to meet increasing student demand. A capital resource of $£ 14.812$ million (a 48 per cent increase on the $1999 / 2000$ quantum) has been allocated to the sector. Of this, $£ 2$ million will be distributed to the Video Services Network, £2 million to local area networks, a further $£ 1$ million to ICTs generally, and $£ 810,000$ to JISC (Joint Information Systems Committee). A sum of $£ 6.02$ million is earmarked for other capital priorities including improving IT equipment and improving ICT infrastructure to enable the sector to play a leading role in the UfI (University for Industry). However, the impetus is clearly a capital one, and there is little or no evidence of strategic or curriculum drives, which presumably are to be left to the colleges themselves.

The number of open-access computers in the colleges surveyed ranged from our given bracket of 6-25 to in excess of 150 . However, there was no significant correlation in the ratio of numbers of computers and numbers of students. For example, one very small residential college had an open-access $\mathrm{PC}$ ratio of 26-75 (our bracket) to 130 full-time students (FTs). The same number of open-access PCs were reported by a college with 2,000 FTs, one with 800 FTs and one with 30,000 students (approximately 7,500 FTs) (Welsh Funding Councils, 1998). Clearly, some students have much easier access to computers than others.

Similarly, no significant relationships were found between the ratio of student and printer numbers or between the ratio of computers and numbers of printers. This tends to indicate that although larger institutions have higher equipment numbers, the student/equipment ratio is not determined by college size but rather by internal resource allocation priorities. Some of the colleges without a clearly designated CAL co-ordinator had large quantities of ICT equipment available on open access, suggesting that there was no relationship between the presence of a co-ordinator and the level of equipment. This was confirmed by a two-sample t-test assuming equal variances. The number of technical staff responsible for maintaining/supporting ICT hardware and software ranged from two in the smallest college to 20 in one of the medium-sized colleges. The student/technical staff ratio ranged 
from 65:1 in the smallest college to a massive 1,579:1 in the largest (figures implying here that college size determined by student numbers is not a reliable indicator of the degree to which modern technology is used).

All the colleges surveyed stated that CAL was used in one way or another in teaching or learning, but to varying extents in the different colleges, extending from the majority of subjects to only one subject. Subjects taught using CAL were diverse, ranging from IT, electronics and automechanics to floristry and sports turf maintenance. Somewhat to our surprise, academic staff were involved in writing or producing material for CAL in almost all institutions, and in some cases in all departments, but in others were more concentrated in subjects such an engineering and IT, even though in certain cases staff in subjects such as horticulture and floristry were also involved in this activity. The material was principally available on an intranet, although some was placed on the Internet, all this tending to suggest that $\mathrm{FE}$ students are acquiring a substantial degree of computer literacy. It appears that a wide range of delivery media is used, including disks, CD-ROM, an intranet and the Internet. However, not unexpectedly given the current move in CAL towards networking, the Internet and an intranet were by far the most commonly used media.

Despite the seemingly high general level of CAL activity indicated by staff involvement, our survey showed that CAL was used only rarely in some institutions, scoring 2 on a scale of 1 to 5 (where $1=$ never, $5=$ at least three times a week). However, in some colleges, including the smallest, CAL was indeed used at least three times a week on average, both as primary teaching medium and for unsupervised use by students outside contact hours. This situation may well be due to the fact that many full-time students are now required to study key skills, something which gives rise to major ICT demands, and to the existence in many FE colleges of networked Flexible Learning Centres. Most FE courses generally (not only in the colleges surveyed) now also have ICT requirements relating to student assignments, for example a requirement that certain assignments be word-processed.

The majority of FE colleges surveyed did not use proprietary Web-based learning tools (seven out of twelve). However, our survey did reveal some use of FirstClass, Blackboard, WebCT, Lotus Learning Space, Hot Potatoes, Discovery and certain Web-based tools which had been developed in-house. On the other hand, three of the colleges not using such learning tools had not even heard of any of the proprietary facilities available, while a further three had heard only of WebCT.

Two-thirds of the colleges surveyed reported having received external funding for CAL purposes, a somewhat disappointing result in the light of so many available initiatives. The funding came from a variety of sources including the BBC, CELTEC, Europe (ADAPT, ERDF, ESF), FEFCW Training and Consultancy Services (TACS), QUILT, Education for Industry (EfI), and the Welsh Development Agency (WDA). QUILT-funded projects in the FE colleges contacted in this study included:

developing multimedia packages to support key skills in communications for vocational students;

developing a college-wide intranet;

delivering key skills to geographically isolated modern apprentices;

developing bilingual (English/Welsh) learning support. 
Half of the colleges surveyed provided at least some distance learning, and among those not currently providing any, one was piloting a scheme, and two others were planning to implement one from September this year. Such a result plainly indicates the growing importance of this type of learning provision, although distance learning at the time of the survey still involved less than 2 per cent of students in the FE colleges surveyed. The most common means for students staying in touch with tutors when not on site was email, followed jointly by telephone and non-email use of the Internet, but in some colleges no communication was reported outside normal contact hours.

Finally, it is worth noting that one of the current spending priorities of FE colleges generally (not only in this survey) appears to be upgrading PCs (a perennial and thorny problem in all educational establishments), improving PC/student ratios (ditto), and networking systems. Colleges have also been waiting for FEFCW to make a strategic move before making investments, a symptom of the rapid development of the new technologies as well as an indicator of the lack of an overall clear policy.

\section{Conclusion}

Although our survey shows, hardly unexpectedly, that the use of ICTs in Welsh FE colleges varies, their significance is undoubtedly growing. The mere fact that all the colleges surveyed used CAL to some extent indicates the broad shift away from more traditional learning and teaching approaches that has taken place in the last decade. Oxtoby (1999) maintains that 'the future of colleges will be dictated by developments in information and communications technology. The impact will be enormous ... in ten or fifteen years' time the student experience will be totally unlike what it is today.' In the current climate, it would be hard to disagree, and our small survey indicates that the shift is indeed taking place. The fact that all colleges were involved in CAL to some extent and, significantly, that academic staff were often involved in the production of the course material is especially indicative.

However, much will depend on increased investment in equipment, which will be forthcoming only if bodies such as the National Assembly for Wales and the UK government turn promises into cash. Our survey has indicated that in some of the colleges the student/equipment ratios are very poor, something which must undoubtedly have an impact on learning and teaching.

Our survey has shown that at present there is little in the way of ILT strategy and structured CAL planning in Welsh FE colleges - the fact that designated IT co-ordinators were only to be found in two-thirds of the colleges and that equipment-student ratios vary widely point to this. In England, access to specifically earmarked ILT funding (which includes ICTs and CAL) is available only if a college has an ILT strategy which takes account of ICT issues. In Wales, such funds do not exist, nor are colleges required to have an ILT strategy. Consequently, ILT and ICT provision is potentially much less structured and funded than in England, and although this may be partially offset in the near future by the availability of European Objective One monies in some areas of Wales, it implies that important opportunities for widening access to and participation in FE may be missed. 


\section{Note}

'The WALES 2000 Project Research Group is funded by an ESF/ADAPT grant. Dr Antje Cockrill is the group's senior researcher. Dr Cliona O'Neill and Mr David Finch are two of the group's researchers. Professor Eberhard Bischoff is the head of the European Business Management School.

\section{References}

Becta (2000), Survey into Information and Learning Technology Provision, Access and Policy in FE Colleges in Wales. Report to the Further Education Funding Council for Wales, Coventry: British Educational Communications and Technology Agency.

Burgess, G.W. (2000), 'Building quality instructional strategies in the design of multimedia courseware', http://gwburgess.home.mindspring.com/Articles/qualteachl.htm.

Clarke, A. (1998), University for Industry IT Sector Modelling, Leicester: National Institute of Adult Continuing Education.

Education and Training Action Group for Wales (1999), An Education and Training Action Plan for Wales, n.p.: ETAG.

Edwards, R., Raggatt, P., Harrison, R., McCollum A. and Calder, J. (1998), Recent Thinking in Lifelong Learning: A Review of the Literature, DfEE Research Brief Research Report No. 80, http://www.dfee.gov.uk/research/re_paper/RR80.doc.

Eisenberg, D. (1998), 'College faculty and distance learning', Virtual University Journal, 1 (2), 82-4.

FEFC (1996), Report of the Learning and Technology Committee (Higginson Report), Coventry: Further Education Funding Council.

FEFC (1999a), Networking Lifelong Learning: On ILT Development Strategy for FE (Circular 99/18), Coventry: Further Education Funding Council.

FEFC (1999b), ILT Implementation Plan (Circular 99/45), Coventry: Further Education Funding Council.

FEFC (1999c), ILT Implementation Plan: Management and Finance (Circular 99/53), Coventry: Further Education Funding Council.

FEFCW (1995), Further Education Funding Council for Wales Bulletin B95/22: FE NET '96, Cardiff: Further Education Funding Council for Wales.

FEFCW (1998), Further Education Funding Council for Wales Bulletin B98/29: Upgrading FE NET Connections, Cardiff: Further Education Funding Council for Wales.

FEFCW (1999), Further Education Funding Council for Wales Newssheet F99/04, September, Cardiff: Further Education Funding Council for Wales.

FEFCW (2000), Further Education Funding in Wales: 2000-2001 (Bulletin B00102), Cardiff: Further Education Funding Council for Wales.

Furnell, S. M., Onions, P. D., Bleimann, U., Gojny, U., Knahl, M., Rder, H. F. and 
Sanders, P. W. (1998), 'A security framework for on-line distance learning and training', Virtual University Journal, 1 (1), 38-47.

Furnell, S., Evans, M. P., Phippen, A. D. and Abu-Rgheff, M. N. A. (1999), 'Online distance learning: expectations, requirements and barriers', Virtual University Journal, 2 (2), 34-43.

Future Unit (1999), Work in the Knowledge-Driven Economy, London: Department of Trade and Industry.

Gain, M. and De Gicco, E. (1999), 'Putting the learning back into ILT', Adults Learning, $11(3), 23-5$.

Gorard, S. and Selwyn, N. (1999), 'Switching on the learning society? Questioning the role of technology in widening participation in lifelong learning', Journal of Education Policy, $14(5), 523-34$.

Gunasekaran, A. and Love, P. E. D. (1999), 'Current and future directions of multimedia technology in business', International Journal of Information Management, 19, 105-20.

Jacobs, G. and Rodgers, C. (1997), 'Remote teaching with digital video: a transnational experience', British Journal of Educational Technology, 28 (4), 292-304.

Jacobs, G. and Rodgers, C. (1998), 'ISDN-based distance learning: tangible costs, intangible benefits', Computers and Education, 31 (1), 41-53.

Jih, H. J. (1996), 'The impact of learners' pathways on learning in multimedia Computer Aided Learning', Journal of Network and Computer Applications, 19, 367-80.

Johnston, R. (1997), 'Distance learning: medium or message?', Journal of Further and Higher Education, 21 (1), 107-22.

Lockitt, B. (1999), Quality in Information and Learning Technology (QUILT) Wales, September 1998 to August 1999, Phase III, Report to the Further Education Funding Council, London: Further Education Development Agency.

Oxtoby, B. (1999), 'Viewpoint: what will FE colleges be like in 2015?', Education and Training, 41 (9), 396-402.

Sargant, N. (1997), 'Technology and adult learners', Adults Learning, 9 (1), 16-23.

Spectrum (1999), Moving into the Information Age - a Regional Benchmarking Study (report produced by Spectrum Strategy Consultants for the Department of Trade and Industry), London: HMSO. The text is also available at http://www.ukonlineforbusiness. gov.uk.

UKERNA (1999), A Study of Networked Video Facilities in Welsh HEIs and FECs and Proposals for a Welsh Video Network, Didcot: United Kingdom Education and Research Networking Association.

Welsh Funding Councils (1998), Further and Higher Education Statistics in Wales, http://www.wfc.ac.uk/stats/statsvol/1997-98/pdfs/stats_vol.pdf.

Welsh Office (1998), Learning is for Everyone, Green Paper, London: HMSO. 